\title{
Can the Inflaton Also Be a Weakly Interacting Massive Particle?
}

\author{
Dan Hooper, ${ }^{1,2,3}$ Gordan Krnjaic, ${ }^{1}$ Andrew J. Long, ${ }^{2}$ and Samuel D. McDermott ${ }^{1}$ \\ ${ }^{1}$ Fermi National Accelerator Laboratory, Theoretical Astrophysics Group, Batavia, Illinois 60510, USA \\ ${ }^{2}$ University of Chicago, Kavli Institute for Cosmological Physics, Chicago, Illinois 60637, USA \\ ${ }^{3}$ University of Chicago, Department of Astronomy and Astrophysics, Chicago, Illinois 60637, USA
}

(Received 17 July 2018; revised manuscript received 4 February 2019; published 6 March 2019)

\begin{abstract}
We propose a class of models in which a stable inflaton is produced as a thermal relic in the early Universe and constitutes the dark matter. We show that inflaton annihilations can efficiently reheat the Universe, and identify several examples of inflationary potentials that can accommodate all cosmic microwave background observables and in which the inflaton dark matter candidate has a weak scale mass. As a simple example, we consider annihilations that take place through a Higgs portal interaction, leading to encouraging prospects for future direct detection experiments.
\end{abstract}

DOI: $10.1103 /$ PhysRevLett.122.091802

Introduction.-Two of the most pressing problems in cosmology concern the unknown physics of dark matter and inflation. Although the existence of dark matter is strongly supported by a variety of observations, the particle identity of this substance remains entirely unknown. Similarly, whereas cosmological inflation is motivated by the flatness and horizon problems [1], and it is supported by the adiabatic and approximately scale-invariant perturbations observed in the cosmic microwave background (CMB) [2-6], we know little about this period of our cosmic history. In this Letter, we consider the possibility that these two seemingly unrelated phenomena are in fact intimately connected. More specifically, we explore a broad class of models in which the field responsible for inflation (i.e., the inflaton) is also a stable particle whose population freezes out of thermal equilibrium in the early Universe to constitute the dark matter. We refer to this scenario as WIMPflation.

The possibility that a single particle could play the dual roles of inflaton and dark matter was mentioned in Refs. [7,8], and studied with more detail in Refs. [9-30]. In particular, McDonald and collaborators [14,17,23] considered a specific and phenomenologically viable model in which the dark matter candidate is also the inflaton, possessing a nonminimal coupling to gravity. In this Letter, our goal is to develop a more general outlook on WIMPflation by identifying the features that are generically required of a model that can simultaneously satisfy all existing constraints pertaining to both inflation and dark

Published by the American Physical Society under the terms of the Creative Commons Attribution 4.0 International license. Further distribution of this work must maintain attribution to the author(s) and the published article's title, journal citation, and DOI. Funded by SCOAP. matter. We present a wide range of inflationary scenarios in which the inflaton can play the role of a thermal relic that also serves as a viable dark matter candidate.

In order for the inflaton to be a thermal dark matter candidate, it must be stable and it must freeze out of equilibrium in the early Universe to yield an acceptable relic abundance. This requires the dark matter to possess interactions that allow it to annihilate with a cross section of approximately $\sigma v \approx 2 \times 10^{-26} \mathrm{~cm}^{3} / \mathrm{s}$ and to have a mass between approximately $10 \mathrm{MeV}$ and $100 \mathrm{TeV}$. Annihilations through these same interactions must take place at the end of inflation and be efficient enough to reheat the Universe to a high temperature. This produces a thermal bath of both standard model particles and inflatons. The weak scale inflatons then proceed to freeze out of equilibrium in the standard way, resulting in the measured abundance of cold, collisionless dark matter.

Inflationary dynamics. - We consider a class of scenarios in which the inflaton $\phi$ is a real scalar singlet whose interactions respect a $Z_{2}$ symmetry, ensuring its stability in the vacuum. The dynamics of $\phi$ are described by the following Lagrangian:

$$
\mathcal{L}=\frac{1}{2} g^{\mu \nu} \partial_{\mu} \phi \partial_{\nu} \phi-V(\phi)+\mathcal{L}_{\text {int }},
$$

where $V(\phi)$ is the inflaton potential and $\mathcal{L}_{\text {int }}$ describes the interactions that enable inflaton annihilation to both reheat the Universe and later to result in an acceptable thermal relic abundance.

For the inflaton to be a viable thermal relic, its mass must lie in the range $10 \mathrm{MeV} \lesssim m_{\phi} \lesssim 100 \mathrm{TeV}$ [31,32]. Thus we adopt an inflaton potential that includes one term to generate the inflaton mass plus a second term to drive inflation (without contributing to $m_{\phi}$ ). Potentials that satisfy these criteria can be written as 


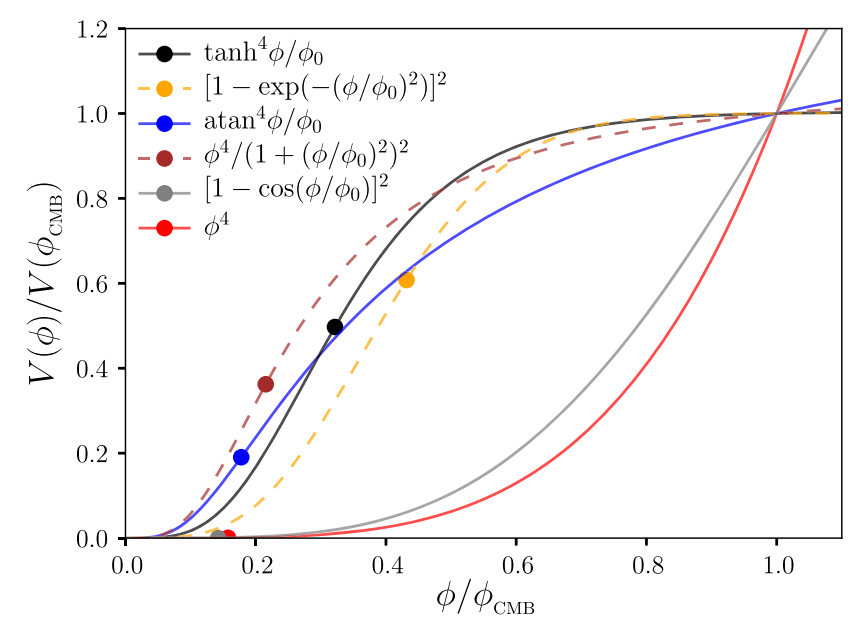

FIG. 1. The inflaton potentials considered in this study are shown here normalized to the value that they take $60 e$ foldings before the end of inflation. The colored dots denote the point at which inflation ends in each model.

$$
V(\phi)=\frac{1}{2} m_{\phi}^{2} \phi^{2}+\lambda \phi_{0}^{4} f\left(\phi / \phi_{0}\right),
$$

where $\phi_{0}$ is a dimensionful constant, the function $f\left(\phi / \phi_{0}\right)$ has a vanishing second derivative at the origin, and thus, $m_{\phi}$ is the inflaton's mass in the vacuum. We consider the following functional forms for the term in the potential that drives inflationary dynamics:

$$
f\left(x=\phi / \phi_{0}\right)=\left\{\begin{array}{l}
x^{4} \\
\arctan ^{4} x \\
\tanh ^{4} x \\
{\left[1-\exp \left(-x^{2}\right)\right]^{2}} \\
(1-\cos x)^{2} \\
x^{4} /\left(1+x^{2}\right)^{2}
\end{array}\right.
$$

The first of these is the $\phi^{4}$ potential, which is the simplest example that meets the requirements listed above. As is well known, however, $\phi^{4}$ inflation predicts a large tensorto-scalar ratio, and is ruled out by modern CMB observations $[5,6]$. We include it here for completeness and for an illuminating contrast with the other potentials under consideration.

Several of the examples described in Eq. (3) are similar to those found in well-known models [33]. The function $\arctan \left(\phi / \phi_{0}\right)$ was originally used in Ref. [34] as a toy example of a potential that leads to a rapidly varying equation of state parameter. The potentials $\tanh ^{4}\left(\phi / \phi_{0}\right)$ and $\left\{1-\exp \left[-\left(\phi / \phi_{0}\right)^{2}\right]\right\}^{2}$ are variations of the $T$-model and $E$-model realizations of the $\alpha$-attractor scenario [35-38]. The potential $\left[1-\cos \left(\phi / \phi_{0}\right)\right]^{2}$ is similar to those found in natural inflation $[39,40]$ but dominated by a higher harmonic [41-43]. Finally, the potential $\phi^{4} /\left[1+\left(\phi / \phi_{0}\right)^{2}\right]^{2}$ arises from the $\phi^{4}$ case in the presence of a nonminimal coupling to gravity of the form $\phi^{2} R[14,17,44]$ and is similar to pure natural inflation [45].

As a paradigmatic example for the interaction term in Eq. (1), we will consider a Higgs portal operator [46,47],

$$
\mathcal{L}_{\text {int }}=-\frac{\kappa}{2} \mathcal{H}^{\dagger} \mathcal{H} \phi^{2},
$$

where $\kappa$ is a dimensionless mixed quartic coupling and $\mathcal{H}$ is the standard model Higgs doublet. In the presence of a background inflaton field, $\phi \neq 0$, the Higgs boson acquires an effective squared mass given by $m_{h}^{2}=-\mu_{H}^{2}+\kappa \phi^{2} / 2$ where $\mu_{H} \sim 10^{2} \mathrm{GeV}$ sets the mass of the standard model Higgs boson at low temperatures. For the models we consider, $m_{h}$ is always much larger than the rate of Hubble expansion during inflation. For this reason, quantum fluctuations of the Higgs field are negligible, and the dynamics of inflation are entirely dominated by the second term in Eq. (2). We emphasize that $\mathcal{H}=0$ is a solution to the classical equations of motion throughout the entire process of inflation: the Higgs can only be "unfrozen" when the phi field value crosses the origin during reheating. Thus, our potential reduces to the case of single-field slow roll for the purposes of inflationary dynamics. Even if $\mathcal{H}$ were initially displaced from the minimum of its potential, such that there is an early phase of multifield inflation, we observe that the $\mathcal{H}$ field has a steeper potential than the $\phi$ field does, and it is reasonable to expect $\mathcal{H}$ will reach the origin while $\phi$ is still slowly rolling. The standard period of single-field slow-roll inflation, driven exclusively by $\phi$, would in this way come to dominate the Universe before reheating.

We emphasize that the second term in Eq. (2) is responsible for the phenomenology of inflation whereas Eq. (4) and the first term in Eq. (2) set the inflaton annihilation cross section and mass. The dark matter and inflationary dynamics are therefore largely modular and independent of one another within this class of models.

If the field $\phi$ is displaced from the minimum of its potential it can drive cosmological inflation and induce the perturbations that we eventually observe in the CMB. For each of the functions listed in Eq. (3), we calculate the slow-roll parameters, which are defined as follows [48]:

$$
\epsilon \equiv \frac{1}{2} m_{\mathrm{Pl}}^{2}\left(\frac{V^{\prime}}{V}\right)^{2}, \quad \eta \equiv m_{\mathrm{Pl}}^{2}\left(\frac{V^{\prime \prime}}{V}\right),
$$

where $m_{\mathrm{Pl}} \simeq 2.4 \times 10^{18} \mathrm{GeV}$ is the reduced Planck mass. Slow-roll inflation occurs when the inflaton field is nearly homogeneous in a Hubble patch and $\epsilon, \eta \ll 1$. We define the end of inflation, $t_{\text {end }}$, as the time when $\epsilon=1$. CMB observables probe the inflaton potential at $\phi_{\mathrm{CMB}}$, which is the value of the inflaton field $N_{\mathrm{CMB}} \approx 50-60 e$ foldings before the end of inflation: 


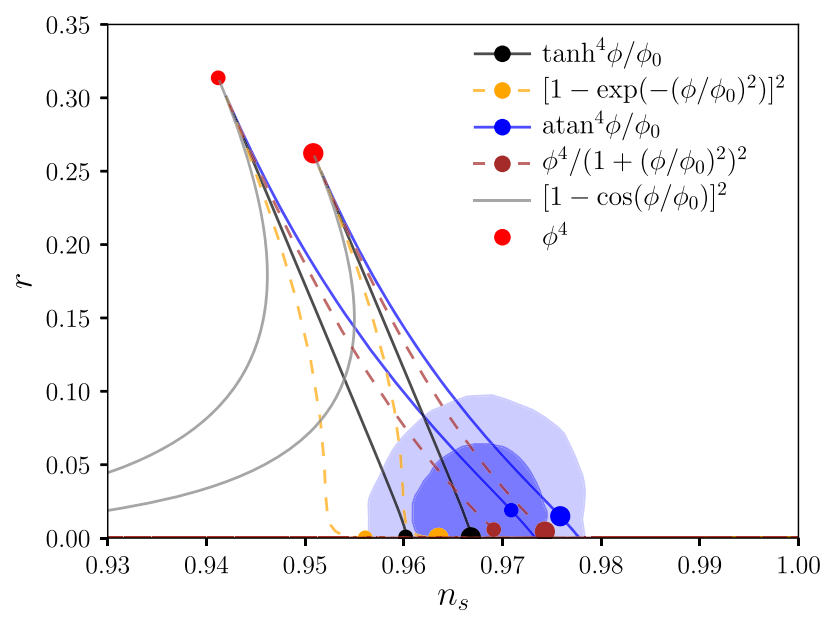

FIG. 2. The tensor-to-scalar ratio $(r)$ and the tilt of the scalar power spectrum $\left(n_{s}\right)$ predicted for each of the inflationary potentials considered in this study. Along each curve, the value of $\phi_{0}$ varies, and the left and right curves correspond to results found for $N_{\mathrm{CMB}}=50$ and 60 , respectively. The colored dots denote the point at which $\phi_{0}=m_{\mathrm{Pl}}$ (no gray point is shown, as $\phi_{0}>m_{\mathrm{Pl}}$ over the entire range shown for this model). Planck measurements (68\% and $95 \%$ confidence contours shown in dark and light blue, respectively) [5] disfavor the $\phi^{4}$ and $\left[1-\cos \left(\phi / \phi_{0}\right)\right]^{2}$ models.

$N_{\mathrm{CMB}}=\int_{t_{\mathrm{CMB}}}^{t_{\mathrm{end}}} H d t=\int_{\phi_{\mathrm{CMB}}}^{\phi_{\mathrm{end}}} \frac{H d \phi}{\dot{\phi}}=\int_{\phi_{\mathrm{end}}}^{\phi_{\mathrm{CMB}}} \frac{V}{V^{\prime}} \frac{d \phi}{m_{\mathrm{Pl}}^{2}}$.

In the last step we have used the slow-roll approximation, $\dot{\phi}=-V^{\prime} / 3 H$.

In Fig. 1, we plot representative examples of the six inflationary potentials being considered in this study, normalized to their value at $\phi_{\mathrm{CMB}}$ and for the case of $N_{\mathrm{CMB}}=60$. For each of these curves, we have adopted a value of $\phi_{0}=m_{\mathrm{Pl}}$, except for in the $\left[1-\cos \left(\phi / \phi_{0}\right)\right]^{2}$ case, for which we show results for $\phi_{0}=10 m_{\mathrm{Pl}}$ (the slow-roll conditions can only be satisfied in this case if $\left.\phi_{0} \gg m_{\mathrm{Pl}}\right)$. The colored dots along each curve denote the point at which $\phi=\phi_{\text {end }}$.

Next, we calculate the values of the tensor-to-scalar ratio, $r=16 \epsilon$, and the tilt of the scalar power spectrum, $n_{s}=1+2 \eta-6 \epsilon$, predicted in this class of models (where $\epsilon$ and $\eta$ are evaluated at $\phi_{\mathrm{CMB}}$ ), and compare these quantities to the constraints imposed by the Planck Collaboration [5]. As can be seen in Fig. 2, the predictions of the $\phi^{4}$ and $\left[1-\cos \left(\phi / \phi_{0}\right)\right]^{2}$ models are in considerable tension with the data. The other four models, however, yield predictions that can easily accommodate the measurements from Planck. In the large $\phi_{0}$ limit, each of these potentials reproduces the $\phi^{4}$ prediction.

For the class of potentials in Eq. (3), we roughly have $V\left(\phi_{\mathrm{CMB}}\right) \sim \lambda m_{\mathrm{Pl}}^{4}$ during slow roll, so the amplitude of the scalar power spectrum satisfies

$$
A_{s}=\frac{V\left(\phi_{\mathrm{CMB}}\right)}{24 \pi^{2} m_{\mathrm{Pl}}^{4} \epsilon} \sim 2 \times 10^{-9}\left(\frac{\lambda}{5 \times 10^{-9}}\right)\left(\frac{10^{-2}}{\epsilon}\right) .
$$

Thus $\lambda$ must be very small in order to accommodate Planck's measurement of $A_{s}^{\text {obs }}=2.196 \times 10^{-9}$ [6].

Naïvely, the Higgs-inflaton coupling in Eq. (4) ruins the shallow slow-roll potential described in Eq. (3) if $\kappa \gtrsim 4 \pi \sqrt{\lambda}$. Specifically, this interaction induces a radiative correction to the inflaton potential, which takes the form $\Delta V=\left(\kappa^{2} \phi^{4} / 64 \pi^{2}\right) \log \left[\kappa^{2} \phi^{2} / \mu^{2}\right]$ at one-loop order. With a judicious choice of $\mu$, we see that $\Delta V$ or any one of its derivatives can be made to vanish at any given field value, but we require the correction to be small over the whole range $\phi_{\text {end }}<\phi<\phi_{\mathrm{CMB}}$, which cannot be accomplished by tuning in this way. For the purposes of this study, however, we only require that $\mathcal{L}_{\text {int }}$ assumes the quadratic form in Eq. (4) for small field values. One way to accomplish this is to generalize the form of the Higgs-inflaton interaction such that the effective coupling is weaker during inflation when $\phi \approx \phi_{\mathrm{CMB}}$ but grows stronger as $\phi$ approaches $\phi_{\text {end }} \ll \phi_{\mathrm{CMB}}$. Such a $\phi$-dependent interaction strength is consistent both with the preceding discussion of inflationary observables and with the following discussion of reheating. Moreover, concrete implementations of interactions that depend on inflaton field value are available in the literature. For example, if the inflaton has a nonminimal coupling to gravity, $\mathcal{L}_{\text {int }}=(\xi / 2) \phi^{2} R$, then the Jordanframe potential takes the form $V=\left[(\tilde{\kappa} / 2) \mathcal{H}^{\dagger} \mathcal{H} \phi^{2}+\right.$ $\left.(\tilde{\lambda} / 4) \phi^{4}\right] /\left[1+\xi \phi^{2} / M_{\mathrm{pl}}^{2}\right]^{2}$ where the inflaton potential reaches a plateau for $\phi \gg M_{\mathrm{Pl}} / \sqrt{\xi}$. This allows $\tilde{\kappa}<$ $4 \pi \tilde{\lambda}^{1 / 2} \sim \mathcal{O}(1)$ while keeping radiative corrections under control (for example, see Refs. [14,35]).

Similarly, the dramatic separation of scales in the inflationary potential $\left(m_{\phi} \ll \phi_{0}\right)$ may give the reader pause. However, as the postinflationary phenomenology is intimately connected to the Higgs sector in our toy model, it is perhaps reasonable to assume that the same as-yetunknown physics that resolves the Higgs hierarchy problem is also responsible for setting the scales in the inflaton sector. We leave deeper exploration of such model-building questions to future work.

Reheating.-As inflation comes to an end, the Universe is filled with an inflaton condensate that transfers its energy to a plasma of standard model particles through the process of reheating [49-52]. In the class of scenarios considered here, a $Z_{2}$ symmetry forbids the inflaton from decaying at late times when $\phi=0$, but this symmetry is spontaneously broken during reheating when $\phi \neq 0$. Consequently, reheating proceeds through a combination of $\phi$ annihilation and $\phi$-dependent decays. Collectively, the nonperturbative description of this evolution is known as preheating [7,8,53-55].

In this section we argue that in the scenarios considered here, reheating efficiently destroys the inflaton condensate, 


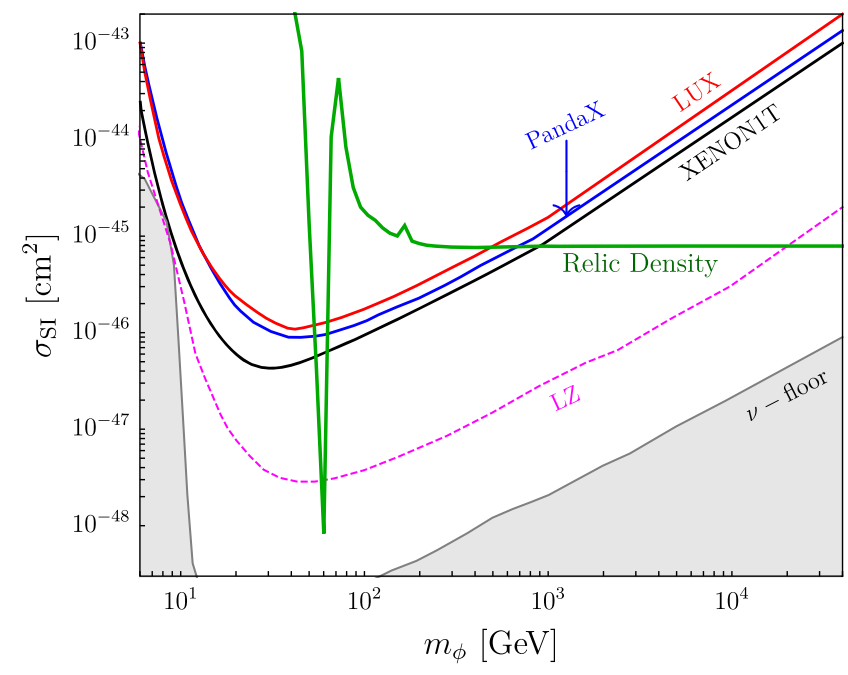

FIG. 3. The spin-independent elastic scattering cross section with nucleons of inflaton dark matter, compared to the constraints from the XENON1T [67], LUX [68], and PandaX-II [69] Collaborations. We also show the projected reach of the LZ Collaboration [70,71], as well as the neutrino floor [72].

generating a high-temperature plasma of standard model particles in thermal equilibrium with inflatons. In the context of the Higgs portal interaction, analytical and numerical studies have shown reheating to be very efficient [17,56-58], easily leading to $T_{\mathrm{RH}} \gg m_{\phi}$. As the dynamics of preheating are highly nonlinear and nonperturbative, a detailed study is beyond the scope of this Letter. In the remainder of this section, we will use a simplified perturbative description of reheating through $\phi$ annihilations to develop the reader's intuition.

Once inflation has ended, the inflaton field begins to oscillate about the minimum of its potential. Since the Higgs mass depends on $\phi$ [see below Eq. (4)], annihilations and decays of $\phi$ are kinematically forbidden for values of $\phi$ for which $V^{\prime \prime}(\phi)<\kappa \phi^{2}$, where $V^{\prime \prime}(\phi)$ and $\kappa \phi^{2}$ are the fielddependent squared masses of the inflaton and Higgs fields, respectively. Higgs production becomes efficient for $|\phi| \lesssim m_{\phi} / \sqrt{\kappa}$, and a burst of Higgs boson production occurs each time $\phi$ oscillates through the origin of the potential [58-60]. The inflaton field reaches the origin on a timescale given by the inverse of the field-dependent inflaton mass, during which Hubble expansion dilutes the energy density by only an order one factor.

To estimate the efficiency of Higgs production, we treat the inflaton condensate as a collection of zero-momentum particles, and we calculate the cross section for the annihilation channel, $\phi \phi \rightarrow \mathcal{H}^{\dagger} \mathcal{H}$. With the Higgs portal interaction in Eq. (4), and for $m_{\phi} \gg m_{h}$, we obtain the following cross section in the nonrelativistic limit

$$
(\sigma v)_{\phi \phi \rightarrow \mathcal{H}^{\dagger} \mathcal{H}}=\frac{\kappa^{2}}{16 \pi m_{\phi}^{2}}
$$

Inflaton annihilations and decays populate the standard model thermal bath, which carries an energy density $\rho_{R}\left(t_{\mathrm{RH}}\right)=\left(\pi^{2} / 30\right) g_{*} T_{\mathrm{RH}}^{4}$. We estimate the annihilation rate of inflatons as $\Gamma \sim n_{\phi} \sigma v$, where $n_{\phi}$ is the density of the inflaton condensate at the end of inflation and $\sigma v$ is given by Eq. (8). Because the condensate is very dense, $n_{\phi} \sim \rho_{\phi} / m_{\phi} \gg m_{\phi}^{3}$, the annihilation rate dramatically exceeds the Hubble expansion rate, $\Gamma \gg H$, once the annihilation and decay channels are no longer blocked. This naïve, perturbative estimate suggests that reheating is very efficient, leading to a rapid transfer of energy from the inflaton condensate to the standard model thermal bath. A more accurate description of reheating would take into account other relevant factors [8], but it is very unlikely that the complete result will differ in some parametrically uncontrolled way from the result in Eq. (8), since $\langle\phi\rangle$ dependent decays give parametrically the same reheating rate as the pairwise $\phi$ annihilations.

We can identify the reheating temperature as follows:

$$
T_{\mathrm{RH}}=\left(\xi \frac{30}{\pi^{2} g_{*}} V\left(\phi_{\mathrm{end}}\right)\right)^{1 / 4},
$$

where $V\left(\phi_{\text {end }}\right)$ is the energy density at the end of inflation, and we have included the quantity $\xi$ to account for the possibility that energy transfer is not perfectly efficient during reheating. From our naïve perturbative calculation, we expect $\xi=\mathcal{O}(1)$ as found, for instance, in instant preheating scenarios [61]. Numerical studies of reheating in other models obtain $\xi \sim \mathcal{O}(0.001-0.1)$ [8,61-63]. In the remainder of this Letter we will assume that the Universe reheats to a high temperature, $T_{\mathrm{RH}} \gg m_{\phi}$, and that the inflaton thermalizes with the standard model plasma.

Dark matter phenomenology.-After reheating, the inflaton abundance follows the equilibrium number density until thermal freeze-out occurs, at a temperature given by $T_{\mathrm{FO}} \simeq m_{\phi} / 20$ [64]. Using the expression for the annihilation cross section given in Eq. (8), including annihilation channels to all standard model particles (and thereby supplemented with the correct kinematic factors at each particle threshold), we find that the inflaton relic abundance is

$$
\Omega_{\phi} h^{2} \approx 0.1 \times\left(\frac{0.3}{\kappa}\right)^{2}\left(\frac{m_{\phi}}{\mathrm{TeV}}\right)^{2}
$$

Thus, the measured dark matter abundance $\left(\Omega_{\mathrm{DM}} h^{2} \simeq 0.12\right.$ [6]) can be obtained for reasonable choices of parameters. This interaction also leads to the following spinindependent scattering cross section per nucleon:

$$
\sigma_{\mathrm{SI}} \simeq \frac{\kappa^{2} v^{2} m_{n}^{2}}{16 \pi m_{h}^{4} m_{\phi}^{2}}\left[\frac{Z}{A} C_{p}+\left(1-\frac{Z}{A}\right) C_{n}\right]^{2},
$$


where $Z$ and $A$ are the atomic number and atomic mass of the target nucleus, $v=246 \mathrm{GeV}$, and $C_{p, n}$ are the effective couplings to nucleons $[65,66]$. For $m_{\phi} \gg m_{h}$ and the case of a xenon target

$$
\sigma_{\mathrm{SI}} \approx 7 \times 10^{-46} \mathrm{~cm}^{2} \times\left(\frac{\kappa}{0.3}\right)^{2}\left(\frac{\mathrm{TeV}}{m_{\phi}}\right)^{2} .
$$

In Fig. 3, we compare this cross section to the constraints placed by the XENON1T [67], LUX [68], and PandaX-II [69] Collaborations. We also show the projected reach of the LZ Experiment [70,71], as well as the neutrino floor below which such experiments encounter an irreducible background from neutrino interactions [72]. Much like other Higgs-portal dark matter scenarios [46,47,73-78], the dark matter in this model is constrained by this class of experiments to approximately $m_{\phi} \gtrsim 900 \mathrm{GeV}$ (except near $m_{\phi} \approx m_{h} / 2$ ).

Although we have focused here on inflaton dark matter with a Higgs portal coupling, we could have considered other interactions to facilitate dark matter annihilation and local dark matter detection. For example, a simple variation could involve inflatons with a mixed quartic coupling with a scalar that is a standard model singlet, allowing the dark matter to annihilate to pairs of "dark Higgses," which then decay to standard model particles. In this variation, direct detection constraints could be significantly relaxed, and thereby allow for lighter inflatons and improved prospects for indirect searches [79-85]. One could also consider models in which the inflaton annihilates to a pair of dark photons or right-handed neutrinos [86].

Summary and conclusions.-In this Letter, we have considered a range of WIMPflation scenarios, in which the inflaton also serves as a viable dark matter candidate. Requiring the inflaton to be stable implies that reheating must be accomplished through a combination of annihilations and background-field-dependent decays. The same annihilation process later sets the thermal relic abundance of inflaton dark matter. We identify several inflationary potentials that can accommodate all current CMB constraints and yield a viable thermal relic abundance of inflaton dark matter. For the case of inflaton dark matter that annihilates through a Higgs portal coupling, we find encouraging prospects for future direct detection experiments.

We would like to thank Mark Hertzberg for helpful discussions. A.J.L. is supported by the University of Chicago by the Kavli Institute for Cosmological Physics through Grant No. NSF PHY-1125897 and an endowment from the Kavli Foundation and its founder Fred Kavli. This manuscript has been authored by Fermi Research Alliance, LLC under Contract No. DE-AC02-07CH11359 with the U.S. Department of Energy, Office of Science, Office of High Energy Physics.
The U.S. Government retains a nonexclusive, paid-up, irrevocable, world-wide license to publish or reproduce the published form of this manuscript, or allow others to do so, for U.S. Government purposes. The views and conclusions contained herein are those of the authors and should not be interpreted as necessarily representing the official policies or endorsements, either expressed or implied, of the U.S. Government or any U.S. Government agency.

[1] A. H. Guth, Phys. Rev. D 23, 347 (1981).

[2] D. N. Spergel et al. (WMAP Collaboration), Astrophys. J. Suppl. Ser. 170, 377 (2007).

[3] M. Tegmark, J. Cosmol. Astropart. Phys. 04 (2005) 001.

[4] L. A. Boyle, P. J. Steinhardt, and N. Turok, Phys. Rev. Lett. 96, 111301 (2006).

[5] P. A. R. Ade et al. (Planck Collaboration), Astron. Astrophys. 594, A20 (2016).

[6] P. A. R. Ade et al. (Planck Collaboration), Astron. Astrophys. 594, A13 (2016).

[7] L. Kofman, A. D. Linde, and A. A. Starobinsky, Phys. Rev. Lett. 73, 3195 (1994).

[8] L. Kofman, A. D. Linde, and A. A. Starobinsky, Phys. Rev. D 56, 3258 (1997).

[9] A. R. Liddle and L. A. Urena-Lopez, Phys. Rev. Lett. 97, 161301 (2006).

[10] V. H. Cardenas, Phys. Rev. D 75, 083512 (2007).

[11] G. Panotopoulos, Phys. Rev. D 75, 127301 (2007).

[12] A. R. Liddle, C. Pahud, and L. A. Urena-Lopez, Phys. Rev. D 77, 121301 (2008).

[13] N. Bose and A. S. Majumdar, Phys. Rev. D 80, 103508 (2009).

[14] R. N. Lerner and J. McDonald, Phys. Rev. D 80, 123507 (2009).

[15] N. Okada and Q. Shafi, Phys. Rev. D 84, 043533 (2011).

[16] J. De-Santiago and J. L. Cervantes-Cota, Phys. Rev. D 83, 063502 (2011).

[17] R. N. Lerner and J. McDonald, Phys. Rev. D 83, 123522 (2011).

[18] A. de la Macorra, Astropart. Phys. 35, 478 (2012).

[19] K. Mukaida and K. Nakayama, J. Cosmol. Astropart. Phys. 03 (2013) 002.

[20] V. V. Khoze, J. High Energy Phys. 11 (2013) 215.

[21] K. Mukaida, K. Nakayama, and M. Takimoto, J. High Energy Phys. 12 (2013) 053.

[22] K. Mukaida and K. Nakayama, J. Cosmol. Astropart. Phys. 08 (2014) 062.

[23] F. Kahlhoefer and J. McDonald, J. Cosmol. Astropart. Phys. 11 (2015) 015.

[24] M. Bastero-Gil, R. Cerezo, and J. G. Rosa, Phys. Rev. D 93, 103531 (2016).

[25] T. Tenkanen, J. High Energy Phys. 09 (2016) 049.

[26] R. Daido, F. Takahashi, and W. Yin, J. Cosmol. Astropart. Phys. 05 (2017) 044.

[27] S. Choubey and A. Kumar, J. High Energy Phys. 11 (2017) 080 .

[28] H.-Y. Chen, I. Gogoladze, S. Hu, T. Li, and L. Wu, Eur. Phys. J. C 78, 26 (2018).

[29] L. Heurtier, J. High Energy Phys. 12 (2017) 072. 
[30] R. Daido, F. Takahashi, and W. Yin, J. High Energy Phys. 02 (2018) 104.

[31] C. Boehm, M. J. Dolan, and C. McCabe, J. Cosmol. Astropart. Phys. 08 (2013) 041.

[32] K. Griest and M. Kamionkowski, Phys. Rev. Lett. 64, 615 (1990).

[33] J. Martin, C. Ringeval, and V. Vennin, Phys. Dark Universe 5-6, 75 (2014).

[34] L.-M. Wang, V. F. Mukhanov, and P. J. Steinhardt, Phys. Lett. B 414, 18 (1997).

[35] R. Kallosh and A. Linde, Phys. Rev. D 91, 083528 (2015).

[36] R. Kallosh and A. Linde, J. Cosmol. Astropart. Phys. 07 (2013) 002.

[37] R. Kallosh and A. Linde, J. Cosmol. Astropart. Phys. 12 (2013) 006.

[38] R. Kallosh, A. Linde, and D. Roest, J. High Energy Phys. 11 (2013) 198.

[39] K. Freese, J. A. Frieman, and A. V. Olinto, Phys. Rev. Lett. 65, 3233 (1990).

[40] F. C. Adams, J. R. Bond, K. Freese, J. A. Frieman, and A. V. Olinto, Phys. Rev. D 47, 426 (1993).

[41] M. Czerny and F. Takahashi, Phys. Lett. B 733, 241 (2014).

[42] R. Kappl, H. P. Nilles, and M. W. Winkler, Phys. Lett. B 753, 653 (2016).

[43] V. Poulin, T. L. Smith, D. Grin, T. Karwal, and M. Kamionkowski, Phys. Rev. D 98, 083525 (2018).

[44] F. L. Bezrukov and M. Shaposhnikov, Phys. Lett. B 659, 703 (2008).

[45] Y. Nomura, T. Watari, and M. Yamazaki, Phys. Lett. B 776, 227 (2018).

[46] J. McDonald, Phys. Rev. D 50, 3637 (1994).

[47] C. P. Burgess, M. Pospelov, and T. ter Veldhuis, Nucl. Phys. B619, 709 (2001).

[48] D. Baumann, Physics of the large and the small, TASI 09, Proceedings of the Theoretical Advanced Study Institute in Elementary Particle Physics, Boulder, Colorado, USA, 2009 (World Scientific, Singapore, 2011), pp. 523-686.

[49] D. Boyanovsky, H. J. de Vega, and R. Holman, Current topics in astrofundamental physics, Proceedings of the International School of Astrophysics $* D$. Chalonge*, 5th Course, Erice, Italy, 1996 (World Scientific, Singapore, 1996), pp. 183-270.

[50] B. A. Bassett, S. Tsujikawa, and D. Wands, Rev. Mod. Phys. 78, 537 (2006).

[51] R. Allahverdi, R. Brandenberger, F.-Y. Cyr-Racine, and A. Mazumdar, Annu. Rev. Nucl. Part. Sci. 60, 27 (2010).

[52] M. A. Amin, M. P. Hertzberg, D. I. Kaiser, and J. Karouby, Int. J. Mod. Phys. D 24, 1530003 (2015).

[53] J. H. Traschen and R. H. Brandenberger, Phys. Rev. D 42 , 2491 (1990).

[54] A. D. Dolgov and D. P. Kirilova, Primordial nucleosynthesis and evolution of early Universe, Proceedings of the International Conference, Tokyo, Japan, 1990 (Kluwer Academic, Dordrecht, 1990), pp. 55-59.

[55] Y. Shtanov, J. H. Traschen, and R. H. Brandenberger, Phys. Rev. D 51, 5438 (1995).

[56] F. Bezrukov, D. Gorbunov, and M. Shaposhnikov, J. Cosmol. Astropart. Phys. 06 (2009) 029.
[57] G. Ballesteros, J. Redondo, A. Ringwald, and C. Tamarit, J. Cosmol. Astropart. Phys. 08 (2017) 001.

[58] K. Enqvist, M. Karciauskas, O. Lebedev, S. Rusak, and M. Zatta, J. Cosmol. Astropart. Phys. 11 (2016) 025.

[59] D. J. H. Chung, E. W. Kolb, A. Riotto, and I. I. Tkachev, Phys. Rev. D 62, 043508 (2000).

[60] M. A. Fedderke, E. W. Kolb, and M. Wyman, Phys. Rev. D 91, 063505 (2015).

[61] G. N. Felder, L. Kofman, and A. D. Linde, Phys. Rev. D 59, 123523 (1999).

[62] J. F. Dufaux, A. Bergman, G. N. Felder, L. Kofman, and J.-P. Uzan, Phys. Rev. D 76, 123517 (2007).

[63] J. M. Hyde, Phys. Rev. D 92, 044026 (2015).

[64] E. W. Kolb and M. S. Turner, Front. Phys. 69, 1 (1990).

[65] M. A. Shifman, A. I. Vainshtein, and V. I. Zakharov, Phys. Lett. 78B, 443 (1978).

[66] M. Cirelli, E. Del Nobile, and P. Panci, J. Cosmol. Astropart. Phys. 10 (2013) 019.

[67] E. Aprile et al. (XENON Collaboration), Phys. Rev. Lett. 121, 111302 (2018).

[68] D. S. Akerib et al. (LUX Collaboration), Phys. Rev. Lett. 118, 021303 (2017).

[69] X. Cui et al. (PandaX-II Collaboration), Phys. Rev. Lett. 119, 181302 (2017).

[70] D. S. Akerib et al. (LZ Collaboration), arXiv:1509.02910.

[71] P. Cushman et al., Proceedings of the 2013 Community Summer Study on the Future of U.S. Particle Physics: Snowmass on the Mississippi (CSS2013): Minneapolis, MN, USA, 2013 (2013).

[72] J. Billard, E. Figueroa-Feliciano, and L. Strigari, Phys. Rev. D 89, 023524 (2014).

[73] M. Escudero, A. Berlin, D. Hooper, and M.-X. Lin, J. Cosmol. Astropart. Phys. 12 (2016) 029.

[74] V. Silveira and A. Zee, Phys. Lett. 161B, 136 (1985).

[75] B. Patt and F. Wilczek, arXiv:hep-ph/0605188.

[76] M. Pospelov, A. Ritz, and M. B. Voloshin, Phys. Lett. B 662, 53 (2008).

[77] J. March-Russell, S. M. West, D. Cumberbatch, and D. Hooper, J. High Energy Phys. 07 (2008) 058.

[78] M. Gonderinger, H. Lim, and M. J. Ramsey-Musolf, Phys. Rev. D 86, 043511 (2012).

[79] M. Escudero, S. J. Witte, and D. Hooper, J. Cosmol. Astropart. Phys. 11 (2017) 042.

[80] J. Liu, N. Weiner, and W. Xue, J. High Energy Phys. 08 (2015) 050.

[81] P. Ko and Y. Tang, J. Cosmol. Astropart. Phys. 01 (2015) 023.

[82] M. Abdullah, A. DiFranzo, A. Rajaraman, T. M. P. Tait, P. Tanedo, and A. M. Wijangco, Phys. Rev. D 90, 035004 (2014).

[83] A. Martin, J. Shelton, and J. Unwin, Phys. Rev. D 90, 103513 (2014).

[84] A. Berlin, P. Gratia, D. Hooper, and S. D. McDermott, Phys. Rev. D 90, 015032 (2014).

[85] D. Hooper, N. Weiner, and W. Xue, Phys. Rev. D 86, 056009 (2012).

[86] J. Alexander et al., arXiv:1608.08632. 